\title{
Furthering Service 4.0: Harnessing Intelligent Immersive Environments and Systems
}

\author{
Anasol Pena-Rios ; Hani Hagras ; Gilbert Owusu ; Michael Gardner \\ IEEE Systems, Man, and Cybernetics Magazine (Volume: 4, Issue: 1, Jan. 2018), pp. 20 - 31
}

\begin{abstract}
:
With the increasing complexity of service operations in different industries and more advanced uses of specialized equipment and procedures, the great current challenge for companies is to increase employees' expertise and their ability to maintain and improve service quality. In this regard, Service 4.0 aims to support and promote innovation in service operations using emergent technology. Current technological innovations present a significant opportunity to provide on-site, real-time support for field service professionals in many areas. It should be no surprise, then, that intelligent immersive environments have the potential to enhance service operations by improving customer service and increasing overall efficiency. Intelligent immersive systems combine immersive technologies with computational intelligence mechanisms to produce adaptive, context-aware environments for advanced decisionmaking support. Such technologies, e.g., mixed reality (MR) and augmented reality $(A R)$, can potentially enhance working environments, optimizing resources by reducing time and location restrictions, leading to much faster knowledge transfer and a deeper understanding of different processes. These methods can also promote faster response times to provide field service technicians with on-the-job assistance in unfamiliar situations.
\end{abstract}




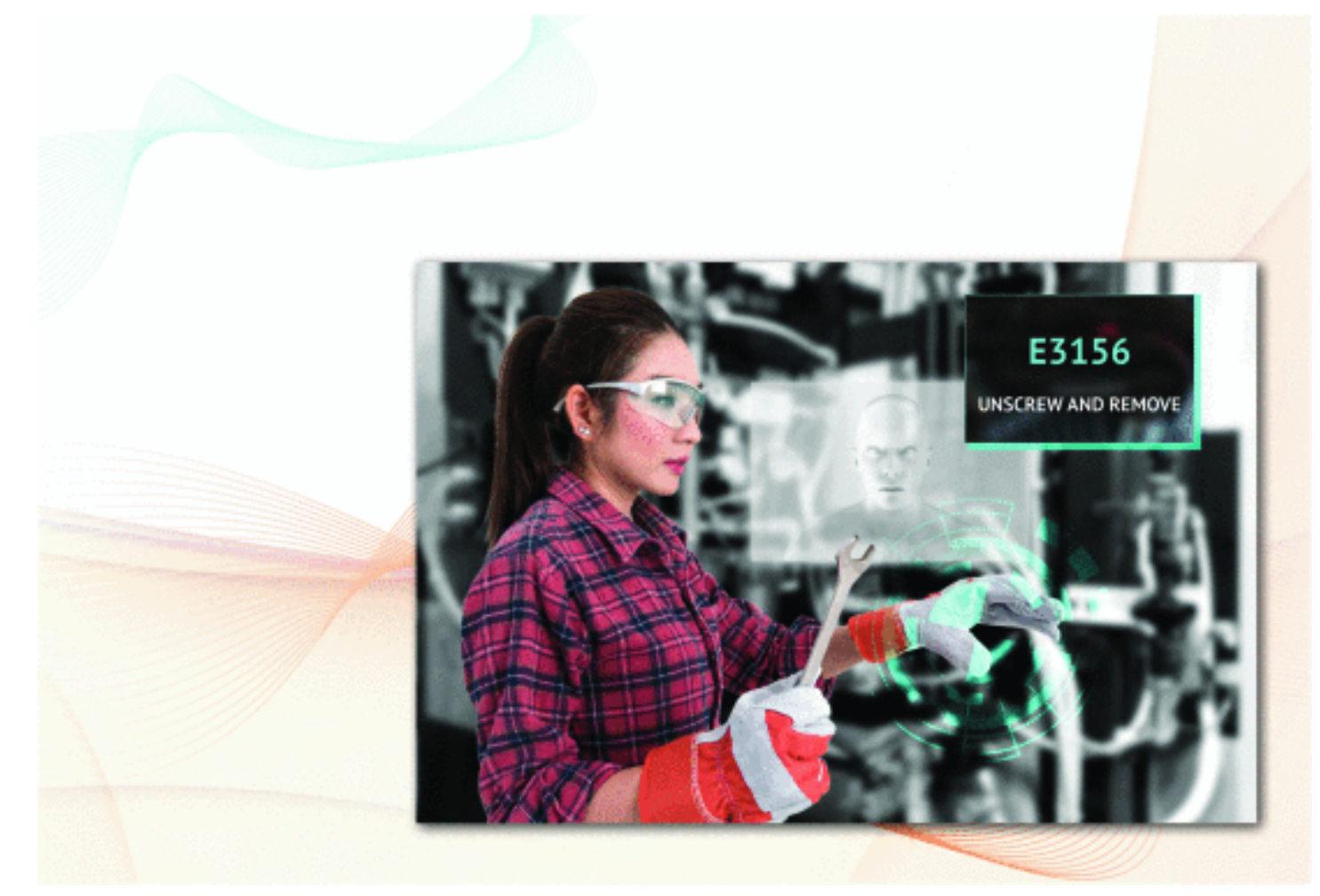

With the increasing complexity of service operations in different industries and more advanced uses of specialized equipment and procedures, the great current challenge for companies is to increase employees' expertise and their ability to maintain and improve service quality. In this regard, Service 4.0 aims to support and promote innovation in service operations using emergent technology. Current technological innovations present a significant opportunity to provide on-site, real-time support for field service professionals in many areas.

It should be no surprise, then, that intelligent immersive environments have the potential to enhance service operations by improving customer service and increasing overall efficiency. Intelligent immersive systems combine immersive technologies with computational intelligence mechanisms to produce adaptive, context-aware environments for advanced decision-making support. Such technologies, e.g., mixed reality (MR) and augmented reality (AR), can potentially enhance working environments, optimizing resources by reducing time and location restrictions, leading to much faster knowledge transfer and a deeper understanding of different processes. These methods can also promote faster response times to provide field service technicians with on-the-job assistance in unfamiliar situations.

\section{The Need for MR in Service Operations}

In recent years, integrating technologies that blur the lines between the digital and physical worlds in the industrial and service sectors has opened up a range of new opportunities, creating the need for human-centered assistance systems to support employees in their jobs. MR can be defined as mechanisms that connect real environments to ones that are completely virtual. AR, which increasingly involves the use of mobile devices and wearable technology, has created growing expectations for its application in real-life scenarios. Initiatives such as the Augmented Reality for Enterprise Alliance (http://thearea.org) and the IEEE Augmented Reality in the Oil/Gas/Electric Industry Group [70] have begun to explore how AR applications might benefit various industries. Market research companies predict that AR technologies will progress from a simple consumer novelty to being integrated into a wide range of commercial applications [1], [2].

AR systems can provide enhanced experiences of real-world situations by overlaying key information and three-dimensional (3-D) visualizations when needed, thus supporting workers' decision-making processes. This can reduce operational costs and improve productivity by leveraging and augmenting 
key work flows and procedures. In contrast, virtual reality (VR) systems present computer-generated simulations that exist separately from the physical world. Due to the synthetic nature of the systems, they may occasionally modify the physical laws governing reality by implementing diverse metaphors (visual, auditory, and haptic) not available in the physical world [3], which makes them ideal domains for conceptual learning and experimentation in controlled environments. One difference between AR and VR is that they achieve different degrees of spatial immersion (or presence). Presence can be defined as the experience of being in an artificially created environment that is realistic enough to make participants feel as though they are actually there [4]-[5][6]. AR's capability to interact with the physical environment makes it more applicable in business and industrial uses.

Although AR has its roots in industrial training, it is presently an emerging technology in both service and industrial applications. The term was coined at the Boeing Corporation by Caudell and Mizell (1992), who introduced a head-mounted prototype to display schematics whose aim was to reduce errors in manual manufacturing tasks, guiding workers on the factory floor. Since then, AR systems have been widely regarded as promising platforms to train and support employees in procedural tasks. For example, repair and maintenance activities can be augmented with 3-D representations of the exact piece of equipment that requires service, showing technicians step-by-step instructions for the process needed to properly restore that piece of equipment to working order. These systems enable hands-on training, providing an engaging experience for employees to interact with the physical equipment and giving them access to data sets and interactive content while immersed in the AR environment. Potential use cases of this technology for the industrial workplace can be broadly divided into the following categories [8]:

- On-the-job support. AR has shown a capacity for mitigating the increasing complexity of industrial maintenance and repair tasks, reducing users' cognitive load by providing support at any time. Additionally, the technology appears to result in a reduced number of unsolved errors [9] and an improvement in the completion time for industrial maintenance tasks [10], thus optimizing resource use.

- Active training. Performing preventive and corrective maintenance tasks while being simultaneously instructed boosts knowledge retention, subsequently improving performance and lowering training costs [11]. Consequently, AR training systems have the potential to be an effective cognitive tool for learning, improving memory recall, and understanding and accelerating the learning curve in real-world scenarios.

- Knowledge and skills transfer. AR could be a powerful tool for reducing training time and accelerating task mastery [11]. However, to achieve this, it is necessary to identify the right scenarios for its use. For example, the technology can provide triggers for key information in maintenance procedures, which not only enables less-skilled workers to competently complete critical tasks but also speeds the employees' transition from novice to expert.

Gartner Research suggests that as technology continues to become more humancentric, AR could bring immersive experiences to the point where communication among people, businesses, and equipment will be thoroughly transparent [12]. The confluence of these technologies aims to augment human capabilities, providing enhanced memory, improved communication, and sharpened senses for multidimensional thinking and problem solving [13] toward the vision of what has been termed the augmented worker. These ideas align with the concept called Service 4.0, which is a collective term for disruptive technologies-e.g., big data, wearables, and AR-that support and promote innovation for service organizations. Service 4.0 is a concept parallel to the term Industry 4.0, which focuses on automation and data exchange in manufacturing technologies based on cyberphysical systems, the Internet of Things (IOT), and cloud computing. The interplay among these technologies and their users occurs either by direct manipulation or with the help of a mediating user interface, which can be created using VR and AR [14].

\section{MR Overview}

Weiser [15] described VR as the concept perhaps "most diametrically opposed" to his vision of ubiquitous computing, in which technology becomes so integrated into everyday physical-world life that it becomes indistinguishable from it. VR, he said, "attempts to make a world inside the computer ... focusing on simulating the world rather than on invisibly enhancing the world that already exists." 
The term VR was first used in 1986 by Jaron Lanier, the founder of VPL Corporation [16]-[17][18], and it is usually defined as "the sense of artificial reality." Thus, VR refers to a highly interactive, computer-generated environment that creates a synthetic setting for users, allowing them to experience a sense of being present in a milieu other than their actual reality by substituting their primary sensory input with data produced by a computer [6], [19]-[20][21].

Weiser's concerns reflect two limitations: 1) the separation that VR imposes on reality and 2) human beings' capability to be present and fully engaged in only one reality at a time. Lifton called this the vacancy problem, i.e., "a noticeable and profound absence of a person from one world, either physical or virtual, while they are participating in the other" [22]. MR tries to solve the challenge of being unable to simultaneously experience the physical and virtual worlds by combining elements of the two in a shared environment. Milgram and Kishino proposed a continuum to represent the different degrees between virtuality and reality, defining anything between the poles as MR [23] (Figure 1).

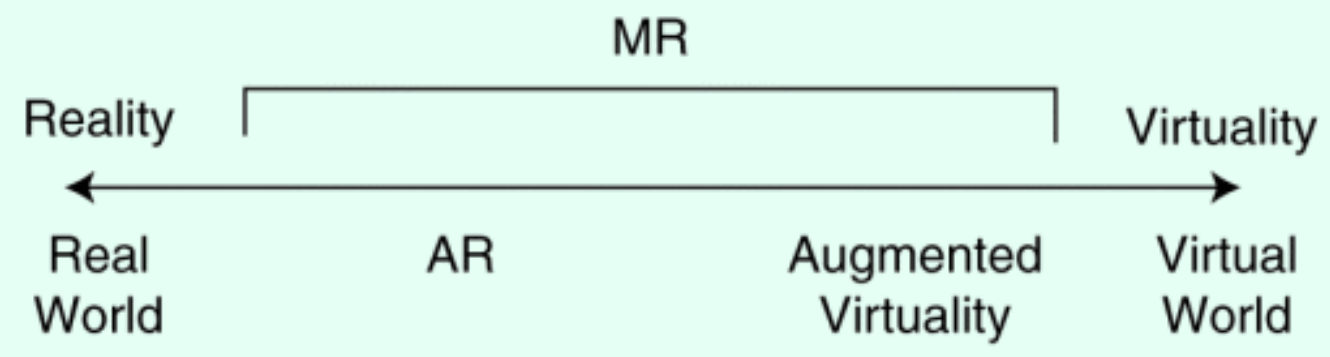

Figure 1. The MR continuum [23].

Within this continuum, AR describes an environment where the physical world is enhanced by adding computer-generated objects using computer-vision methods to make them appear as if they coexist in the same dimension [24]. Therefore, AR supplements reality rather than completely replacing it. AR displays useful information that is not directly detected by the senses, helping users to perform realworld tasks and facilitating their understanding of complex scenarios [25]. Its potential relies on the possibility of enhancing reality and making invisible things visible [24]. Azuma et al. identified three main technologies that support AR systems, which can be considered for any MR system in general [25]:

1. Interaction technologies. These enable the user to manipulate virtual elements in real time. They include the use of tangible interfaces, such as touch screens and haptic devices, and natural interaction interfaces, such as gesture and speech recognition.

2. Display technologies. These allow the positioning of real-world elements with computergenerated objects and information, superimposing them on the user's field of view. These technologies can be classified as wearable technology (e.g., head-mounted displays and smart glasses), handheld displays (e.g., mobile devices like tablets and smartphones), and fixed projective displays (e.g., stationary screens and caves) [8], [26]. While fixed projective displays do not offer the immersive experience of wearable devices or the portability of handheld displays, they are a low-cost alternative.

3. Tracking technologies. These methods make possible the illusion of a true blend between virtual elements and the real world. For example, in a transparent AR display, it is very easy for the human eye to perceive any mismatch between real objects and virtual graphics, dashing the illusion of coexistence [27]. Accuracy in matching, therefore, is quite important. Tracking is the activity of locating the user's position and orientation in reference to an environment [28]. It is generally based on two approaches: 1 ) the use of computer-vision techniques and 2) the use sensor devices. AR tracking, however, has moved from simple marker-based systems to natural-feature tracking and hybrid sensor-based methods [27]. Within sensor tracking, it is possible to identify two main scenarios: 
- Outdoor tracking is generally based on global positioning system (GPS) hardware, which is used to provide location over a wide area. The GPS is a navigation system based on satellite information, owned and maintained by the U.S. government, and accessible to anyone with a GPS receiver. The GPS's accuracy depends on multiple factors, including atmospheric effects, sky blockage, and receiver quality [29].

- Indoor tracking uses inertial-motion devices, such as accelerometers and gyroscopes, which are available in mobile and wearable devices. Inertial tracking is faster and more robust when rapid changes occur compared to vision-based tracking methods [30]. A downside of inertial trackers is that they tend to drift due to noise accumulation.

Furthermore, errors in tracking systems can be present because of the different levels of accuracy among sensors associated with dynamic environments, which are affected by environmental noise and sensor wear and tear. A common approach to overcome these issues is the fusion of multiple devices to increase the accuracy of the tracking and minimize its errors.

As Weiser predicted, technology is gradually disappearing from our consciousness as it becomes integrated in our everyday lives [15]. Immersive technologies have become more pervasive, thanks to mobile and wearable devices, generating large amounts of contextual information. This creates the opportunity to implement artificial mechanisms for accurate context representation, including for adaptation and learning.

\section{Intelligent Immersive Systems for Service Operations}

As previously described, Service 4.0 aims to support and promote innovation in service operations for organizations using emergent technologies. Service can be understood as economic activities that satisfy consumers' needs but produce no tangible goods [31]. From this perspective, several challenges can be identified:

- the increasing complexity of automatized technology components, which can include multiple distributed, configurable components, adding complexity to operational systems

- workers' increased mobility, particularly for service operations, in which staff members usually need to travel to different locations for maintenance and supply tasks

- high uncertainty levels present in real-world applications due to diverse factors, such as the environment and human behavior.

To meet these challenges, we should consider mobile, context-aware systems to provide users with relevant information and services retrieved from companies' databases, tailoring their application through gathered contextual information. Context could be defined as any information that can be used to characterize an entity's situation, an entity being a person, place, or object that is considered relevant to the interaction between a user and an application, including the user and the application itself [32]. Therefore, contextual information in the workplace can be detected with the help of sensors, wearable devices, indoor and outdoor positioning systems, and object recognition capabilities (using fixed or wearable cameras), all of which can collect historical data for further analysis.

Furthermore, information about equipment used by the worker can be collected via smart objects and IoT devices. IoT accessories are capable of identifying other devices by sending information among themselves based on standard and interoperable communication protocols, creating a dynamic global network infrastructure with self-configuring capabilities. Additionally, natural communication between the user and such systems can be provided via immersive technologies, combining visual information with natural interaction, using AR, gesture recognition, haptic devices, and dialogue-driven voice control. Figure 2 illustrates this structure. 


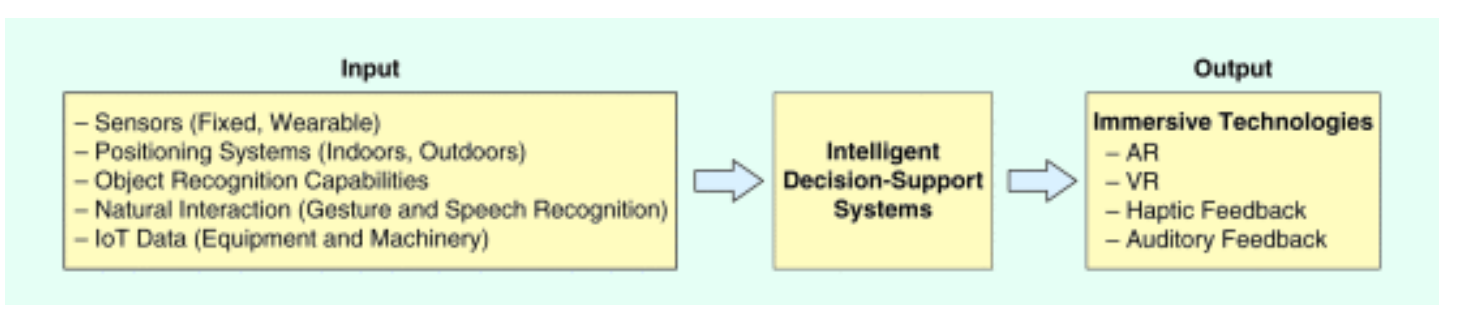

Figure 2. An intelligent immersive decision-support system.

Many technologies can be used to gather contextual information. But to create adaptive immersive systems, it is necessary to combine these methods with computational intelligence techniques to create intelligent applications that are responsive to users' needs and behavior. Intelligent immersive decision-support systems can be used not only to assist decision making, providing recommendations for situations based on real-time contextual information, but also as hands-on training platforms for active training.

A decision-support system can be defined as an adaptive system that aids in solving nonstructured problems using models and data that usually are collected from end users in an interactive and iterative process, generally including a knowledge component [33]. These systems usually have three basic characteristics [14]:

1. They support the worker in the best way possible in all types of situations, particularly in unexpected eventualities.

2. They provide a dynamic perception of the situation that is adaptive to an employee's actions in real time.

3. They are based on knowledge assets that are intuitively and cost-effectively generated from existing company data and constantly upgraded.

Thus, the generation of relevant solutions supposes a joint process of building context by means of the user and the system working as a team. Human-machine teaming focuses on the explicit allocation of cognitive functions and responsibilities between a human and an artificial system to achieve specific goals [34]. From this perspective, human and machine intelligence-while inherently different and not interchangeable — can be seen as complementary [35]-[36][37]. Therefore, tasks are not executed based only on human action or machine performance; rather, the aim is for them to be shared and implemented by both parties working in sync [38], [39].

Hence, it is important to consider other aspects of human-machine teaming, such as humanmachine interaction, communication, and share of the cognitive load. Combining immersive technology with a decision-support system could help with these issues, as it would provide a natural interaction between the user and the system that could lead to better human-machine interaction and communication. Here, immersive technologies could help in the creation of what can be called a virtual assistant focused on domain-specific content.

Finally, as interaction occurs with real-life equipment used on the job, an intelligent immersive decision-support system presents engaging opportunities for active training, reinforcing sensorimotor skills, memory, and higher-order thinking skills used in problem-solving scenarios. Workers could benefit from curated content presented in an immersive way, such as 3-D visualizations of specialized equipment, immersive representations of factory floors and warehouses, or step-by-step instructions with graphical demonstrations overlaid on the physical equipment. Some research studies suggest that industry-oriented AR applications, in particular, have the potential to support users on the job and enhance learning, improving performance and lowering operational costs [11], [40]. Learning with technology (rather than from technology) is what distinguishes technology as a cognitive tool [41]. Moreover, learning within technology creates a mechanism of interaction between content and 
experience [42], [43]. Immersive learning can provide highly interactive first-person sensory scenarios, creating unique real-life learning experiences in the work field.

\section{Case Studies}

In previous works [44], [45], we introduced examples of intelligent immersive systems for advanced decision-making support, which combine fuzzy logic with immersive technologies to provide field service technicians with assistance when they face new challenges. These solutions aim to improve customer service by promoting faster responses and to optimize resources by reducing the restrictions of time and location.

Fuzzy logic attempts to mimic human thinking; it plays an important role in modeling and representing imprecise linguistic human concepts, such as "close" and "far," performing well despite the uncertainty, noise, and imprecision in real-world settings [46]. Fuzzy logic extends classical set theory to calculate intermediate values between true and false, providing a smooth transition when encountering uncertainties. In addition, it uses linguistic IF-THEN rules to model behavior in a human-readable form. Fuzzy logic approaches for learning and tracking users' behavior have been employed in different scenarios [47], [48].

Type-2 (T2) fuzzy sets were conceptually introduced by Zadeh [49]. While type-1 (T1) fuzzy sets do well managing short-term uncertainties (e.g., imprecision associated with sensors and actuators, or slight user-behavior changes), T2 fuzzy sets are designed to model and handle higher levels of uncertainty, such as those present in real-world environments. This is because T1 fuzzy sets use precise and crisp membership functions, whereas T2 fuzzy sets use fuzzy membership functions (i.e., the membership value for each element is a fuzzy set) instead of a crisp number-as in $[0,1]$. Thus, T2 fuzzy sets provide two degrees of freedom via the footprint of uncertainty and the 3-D nature of these sets [50].

Figure 3(c) shows the structure of a T2 fuzzy logic system [52], in which crisp inputs are first fuzzified, converting them to input T2 fuzzy sets. Then, the inference engine identifies the rules fired from a previously defined rule base, combining them to produce output T2 fuzzy sets. Then, the T2 fuzzy output sets are reduced, mapping them to T1 fuzzy sets (also known as type-reduced sets) by combining them and performing a centroid calculation. Finally, the type-reduced sets are defuzzified (by taking the average of the type-reduced set) to obtain crisp outputs [52]. In our study case, we used an interval T2 fuzzy logic system to simplify the computation (as opposed to a general T2 fuzzy logic system) by setting all of the third-dimension values equal to one [51]-[52][53]. This makes it more suitable for real-time applications. An interval T2 fuzzy set is illustrated in Figure 3(d). Next, we present two specific implementations using immersive technologies and fuzzy logic toward the creation of intelligent immersive systems for service operations. 


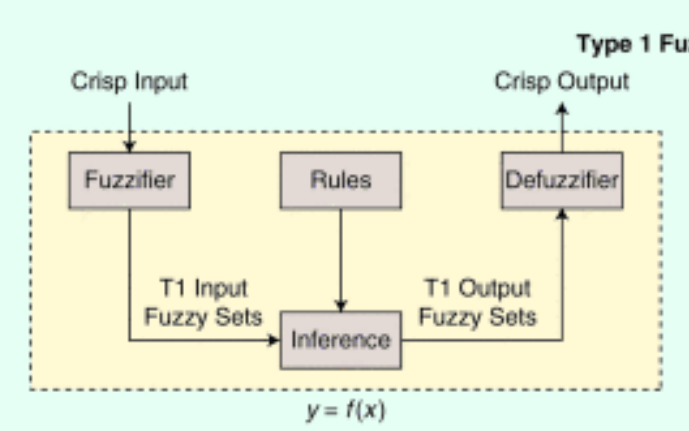

(a)

Type 2 Fuzzy Logic

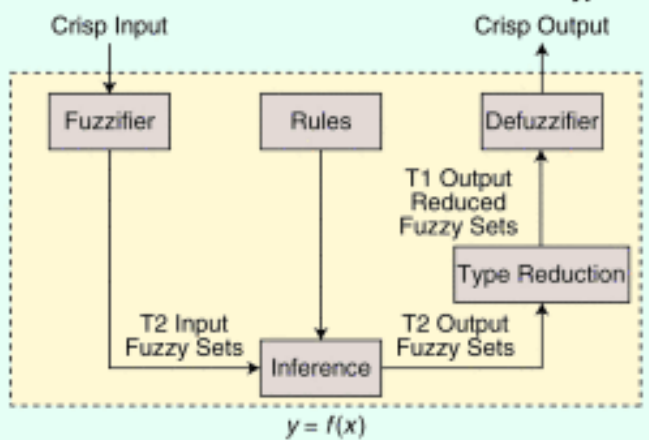

(c)

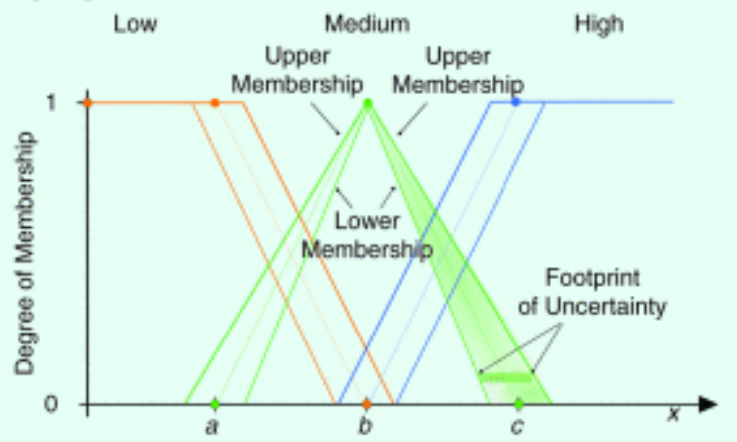

(d)

Figure 3. (a) The structure of a T1 fuzzy logic system [51], (b) a T1 fuzzy set, (c) the structure of a T2 fuzzy logic system [52], and (d) an interval T2 fuzzy set.

In [44], we introduced a novel system using immersive technology and fuzzy logic for equipment monitoring and indoor user tracking (Figure 4). The system was designed to support service operation technicians in three different scenarios:

1. remote equipment monitoring and control, via a VR environment

2. on-the-job monitoring, using an AR application (app)

3. remote expert support, via an MR environment. 


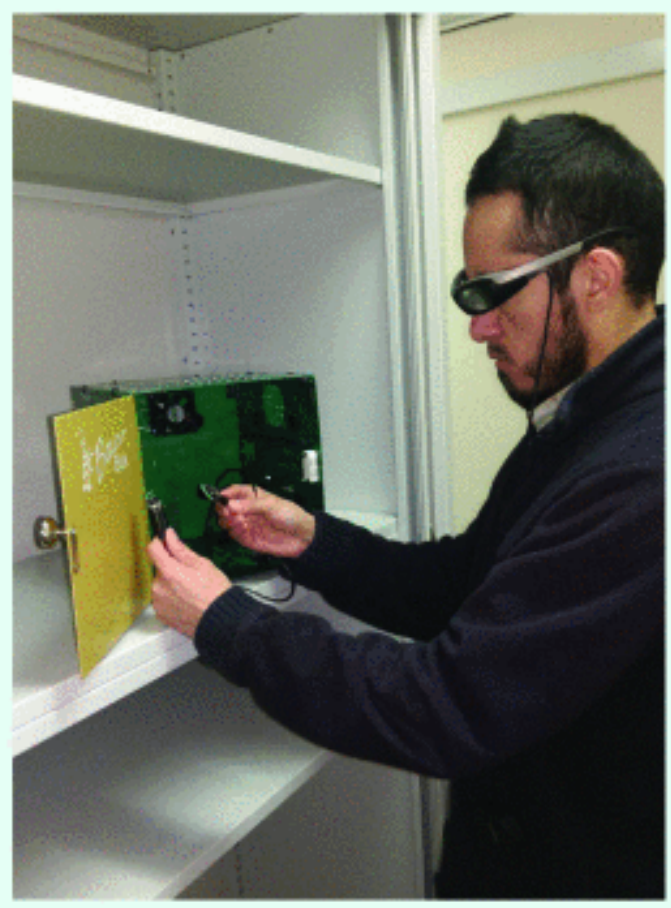

(a)

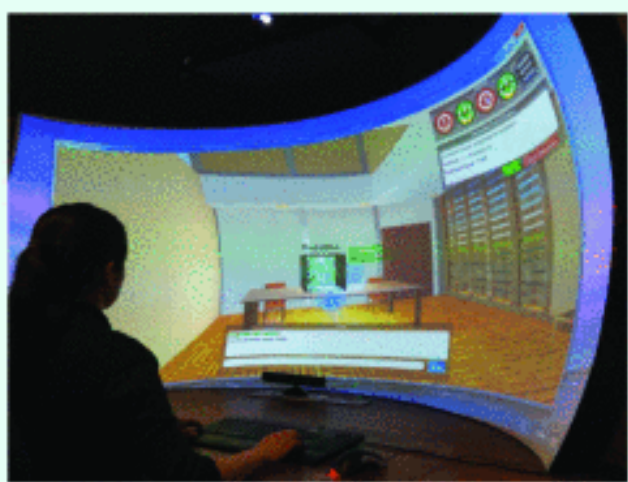

(b)

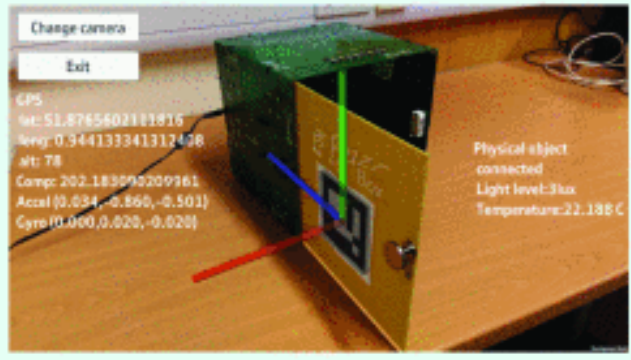

(c)

Figure 4. An MR monitoring system. (a) A technician, (b) an immersive VR control center, and (c) a first-person view of the AR app.

Using a T1 fuzzy logic controller, the system controlled changes in sensors and actuators in the physical equipment and represented them in the VR environment, using 3-D versions of the equipment. This achieved bidirectional communication between the physical and virtual objects in real time. Synchronization between physical objects and virtual objects creates mirrored objects (also known as digital twins) that exist in both worlds simultaneously. This real-time synchronization is defined as a dual-reality (or cross-reality) state [54]. While all hardware objects have digital twins, software objects do not necessarily need twins (twinning is primarily a mechanism to synchronize virtual and physical versions of the same component). This allowed technicians to monitor remote equipment, changing configurations when needed, with the benefit of seeing the effects of the changes in real time using the 3-D representation. Thus, when a technician is needed on site, an AR app could show sensor data to the worker in real time, providing supplementary information when a specific device is under repair.

Finally, using an adaptive fuzzy-based Kalman filter, the system allowed indoor user tracking based on information collected from the inertial measurement unit sensors on wearables (i.e., a mobile phone and head-mounted display), calculating the technician's position and rotation. This information can be useful in situations where a technician needs extra support from an expert. Here, the expert could log into the VR environment and immediately have access to the equipment status and technician position to provide guidance based on an accurate simulation of the technician's view.

In [55], we presented a system to locate specific company equipment using private maps and outdoor positioning sensors (Figure 5). Here, the system captured an engineer's geolocation and orientation, based on GPS and compass sensor information taken from a mobile device. This information was validated by a software agent that compared the worker's previous location with the current one. Then, the agent calculated the difference between the user's current position and the asset's position, obtaining the coordinates (latitude and longitude) of the goal asset from a private repository. This 
difference was used as the input for an interval T2 fuzzy logic system, which provided two outputs: direction and movement. Next, the software agent converted fuzzy outputs to human-understandable directions, which were sent to a head-mounted display used by the engineer, who could hear the directions using text-to-voice functionality and see visual aids to help him reach the asset's location. These outputs were updated in real time as the user kept moving until reaching the goal.

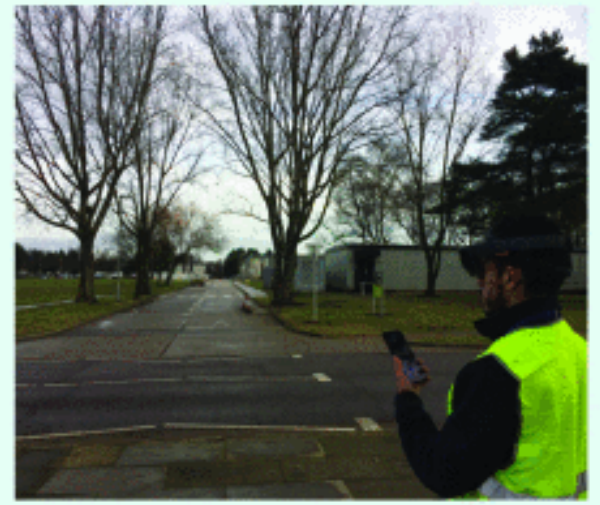

(a)

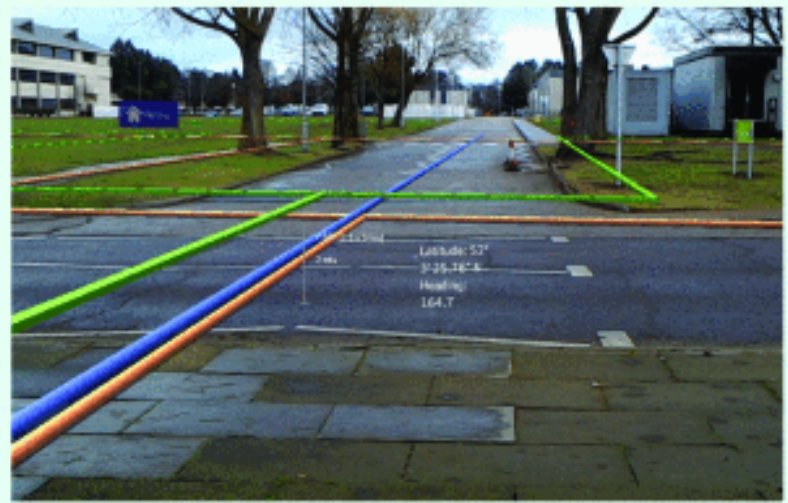

(b)

Figure 5. An intelligent immersive assistant system [45]. (a) An engineer and (b) a first-person view of the AR app.

\section{Intelligent Wearables in Service Operations}

'The case studies previously mentioned rely on the use of intelligent wearables, which can be defined as wearable devices with context-aware capabilities that allow more immersive user-centered interactions. They provide an extra advantage to increase efficiency in service operations. For example, options such as head-mounted displays and smart glasses allow operators' hands to be free, enabling them to work in the physical world and access desired information or services at their moment of need, thus empowering immediate, contextual usage. Broadly speaking, smart glasses are wearable AR devices that capture and process a user's physical environment and augment it with virtual elements [56]. Other intelligent wearables include advanced voice recognition, real-time modeling of 3-D spaces, ever-faster connection speeds (e.g., fifth generation), and artificial intelligence mechanisms. Their purpose is to aid in imitating real-world interactions, focusing on adapting to the user and situation instead of the other way around.

Market research companies predict significant growth in the use of intelligent wearables, identifying them as a priority for technology organizations and business leaders [1], [2]. These technologies are able to connect the physical and digital worlds in new ways by using adaptive algorithms that allow systems to learn from in-the-field data and thus accumulate practical knowledge, which can hardly be gained in a controlled test environment. Using such methods, specialized service staff persons develop an intangible and invaluable body of knowledge cultivated from years on the job. Digital documentation prevents this priceless information from leaving the organization (e.g., when an expert employee changes roles within or outside the organization, or when a senior engineer retires). Today, experiential knowledge is rarely documented within the workplace [57]. But intelligent wearables have the potential to bridge the knowledge and skills gap by using the know-how of expert engineers to construct content for service operations, extending the availability of this information to all workers and achieving transfer of field knowledge and skills.

However, it is important to consider that solving workplace problems using intelligent wearables is greatly dependent on linking these devices to companies' back-end systems, where predictive analytics can proactively anticipate user needs and support intelligent immersive user experiences. For example, such systems could assist technicians with a piece of equipment's repair history, 
provide employees with step-by-step guidance for a particular task, augment human capabilities by providing workers with a sort of X-ray vision to show machinery parts and installation topologies, and bring users' attention to problematic sites. In addition, wearables could support remote interaction with and feedback from experts through integrated videoconferencing and collaboration tools.

Companies are already generating specialized platforms for key use cases. One instance is aerospace manufacturer Boeing, which is launching a new venture company called HorizonX, in partnership with the enterprise AR software firm Upskill, to connect its applications to AR headsets for their front-line workers. Boeing is deploying this initiative across multiple locations for its manufacturing plants, maintenance and repair facilities, and distribution centers. One pilot project at a wiring harness assembly operations facility has achieved early results of a $25 \%$ rate of improvement in production time and better-quality work [58]. Such progress could increase the quality of customer service by allowing employees to do their jobs more effectively and by developing an enhanced insight into employee activities.

\section{Challenges of Intelligent Immersive Systems in Service 4.0}

While having promising potential, intelligent immersive technology and systems also present several challenges before they can enter the mainstream in service operations and industrial applications. For example, smart glasses and AR-based technology present a number of practical challenges for implementation in the field, such as portability, ruggedness, field of vision, visual occlusion limitations, ergonomics, environmental noise (e.g., light) interference, battery duration, and lack of ubiquitous high-speed Internet connection. Thus, to support service operations, these technologies need to become cost effective, scalable, and easily reproducible [59]. Although coming down in both price and size, smart glasses remain expensive and bulky.

We conducted a small user evaluation with telecommunications engineers from Ipswich and Newcastle in the United Kingdom in different sessions between December 2016 and February 2017 on the perceived effectiveness and usability of one-to-one real-time support using intelligent wearables. The sample consisted of five males and two females ranging in age from 20 to 56 (mean = 32). All of the participants were familiar with computers. We used a Microsoft HoloLens device, which is a full AR-enabled untethered wearable computer with transparent holographic lenses combined with spatial mapping, voice, speaking, and gesture recognition [60]. The focus was to evaluate the way people perceive, accept, and adopt technology for the tasks it is designed to support. The results from the evaluation showed that participants thought that the equipment was generally easy to use and helpful in completing their assigned tasks (Figure 6). Disadvantages of the hardware were related to the reduced field of view and the device's weight and cost. Initiatives such as the IEEE Smart Glasses Roadmap Group [61] are working to promote and facilitate the adoption of smart glasses in several markets and applications, and it is likely that technology developments will soon address the limitations. 


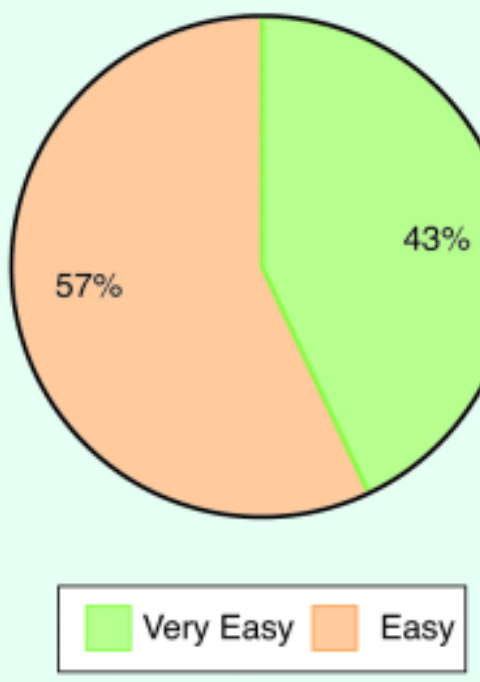

(a)

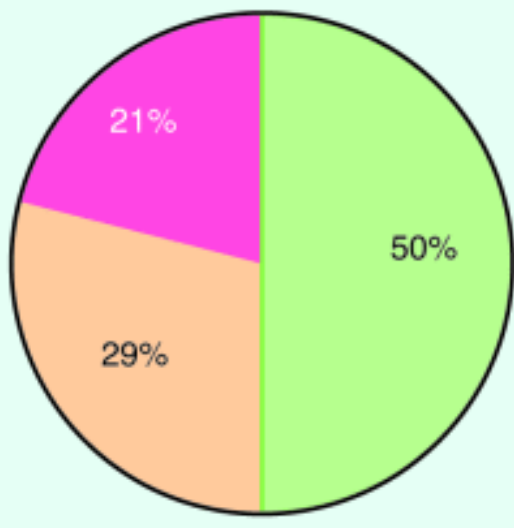

Strongly Agree
Agree

(b)

Figure 6. The results from the Microsoft HoloLens user evaluation. (a) Answers to the question, "How easy or difficult did you find it to complete the activity assigned using the equipment?" (b) Reactions to the statement, "It was helpful to use the equipment in my assigned tasks."

Another reason that the implementation of intelligent immersive systems remains limited is in large part due to the expense associated with creating and changing content to improve procedures in everyday business operations [62], [63]. Content creation software is limited, as content is usually not generated in real time, and it lacks flexibility and ease of integration. For example, AR authoring is a time-consuming procedure that involves creating 3-D models, adding texture, and positioning to create overlays. Creating or modifying AR content requires expertise in graphics and 3-D modeling, adding an extra layer of difficulty for authoring and modifying content. Moreover, content designers are typically not engineering experts. To be relevant, the content needs to be designed on a model focused on a system's core domain and domain logic. Domain-driven design [64] is an approach to developing software for complex needs by deeply connecting the implementation to an evolving model of the core business concepts. Intelligent immersive systems could collect initial data to be used by automatic or semiautomatic content generation, allowing technical and domain experts to iteratively validate the content avoid oversimplification and nonrelevant information. For example, in [65], the authors presented a context-aware AR system for maintenance tasks that allowed users to author content. A preliminary qualitative evaluation of this bidirectional-authoring AR system showed its usability in supporting maintenance technicians on the job, reflecting the value of user-generated content.

Another challenge for intelligent immersive systems is to allow field service technicians to work not only reactively but proactively. An anticipatory system would be one that 1) uses knowledge about future states to decide what action to take at the moment and 2) contains a model of itself and the relevant part of its environment and is able to use this model to anticipate the future. The system would then determine its behavior according to its predictions (i.e., it allows an expected future state to affect its present state). This implies that an intelligent system must be equipped with a simulation component.

As discussed in previous sections, intelligent immersive systems can be used as a cost-effective training platform, providing attractive content and giving new life to traditional methods. Because some technologies use unfamiliar concepts, additional training time is usually required. To be effective in an industrial setting, intelligent immersive systems for training need to be easy to use, 
based on users' vocabulary, compatible with existing technologies, and of reasonable cost [66]. With many attractive benefits, one potential disadvantage of training using immersive technologies is the risk of trainees developing an overdependence on the technology. To compensate, training should scaffold learning and check for understanding to prevent an overreliance on the guidance of the visual clues, auditory aids, and other haptic feedback so that the engineers will be able to perform the maintenance activities without the technology [67].

A final challenge for the effective integration of new technologies is linked with barriers to success. These barriers fall primarily into two categories: 1) technology and infrastructure and 2) human use and adoption [68]. The more complex and difficult of these two is human resistance to change with adoption of new technology. Innovation cycles for new technologies have become shorter and shorter. Implementing technology requires change, which humans tend to resist. While the adoption of a new technology may be of considerable potential benefit to an industry, employees do not necessarily hold the same view of a technology's promise, regardless of any research showing the opposite. For example, feedback from our HoloLens user evaluation raised a concern about the equipment's sophistication possibly posing an obstacle to some engineers, particularly those in the higher age range or those not so enthusiastic about technology.

Haymes [69] developed a three-point approach to overcoming resistance to technological change in education that could also be applied to industrial and service operations.

The key elements of his Three-E Strategy are as follows:

1. Evident. Workers need to be aware of the technology and expect it to be useful.

2. Easy to use. The technology needs to be intuitive to use.

3. Essential. It must become essential to workers and productivity.

Fundamental to framework success is the implementation of an effective feedback loop. The implementation of industry innovation can face many challenges. Resistance to change can be a significant factor in the outcome of a business innovation process. Implementing intelligent immersive technology in the workplace requires substantive changes in policies and procedures. While not all employees resist technological innovation, resistance to change in the workplace is not uncommon.

\section{Conclusion and Future Work}

The service operations field is complex and knowledge intensive. It can benefit from the use of intelligent immersive environments and systems to leverage processes and tasks for field service employees. (Figure 7 summarizes the main benefits of an intelligent immersive system for service operations.) Such systems can provide crucial decision-making support to assist field service engineers by providing information for accomplishing specific job-related tasks (e.g., supply, repair, maintenance, and construction work), using intelligent wearables combined with computational intelligence techniques. They can promote faster solutions to everyday tasks, improving the customer experience, reducing restrictions of time and location, and optimizing resources. Additionally, they can improve the skills transfer process, capturing knowledge in real time and empowering field service professionals. They can place experts' knowledge at the disposal of a distributed workforce, reducing the risk of delays in service due to errors caused by lack of familiarity with procedures and tasks. 


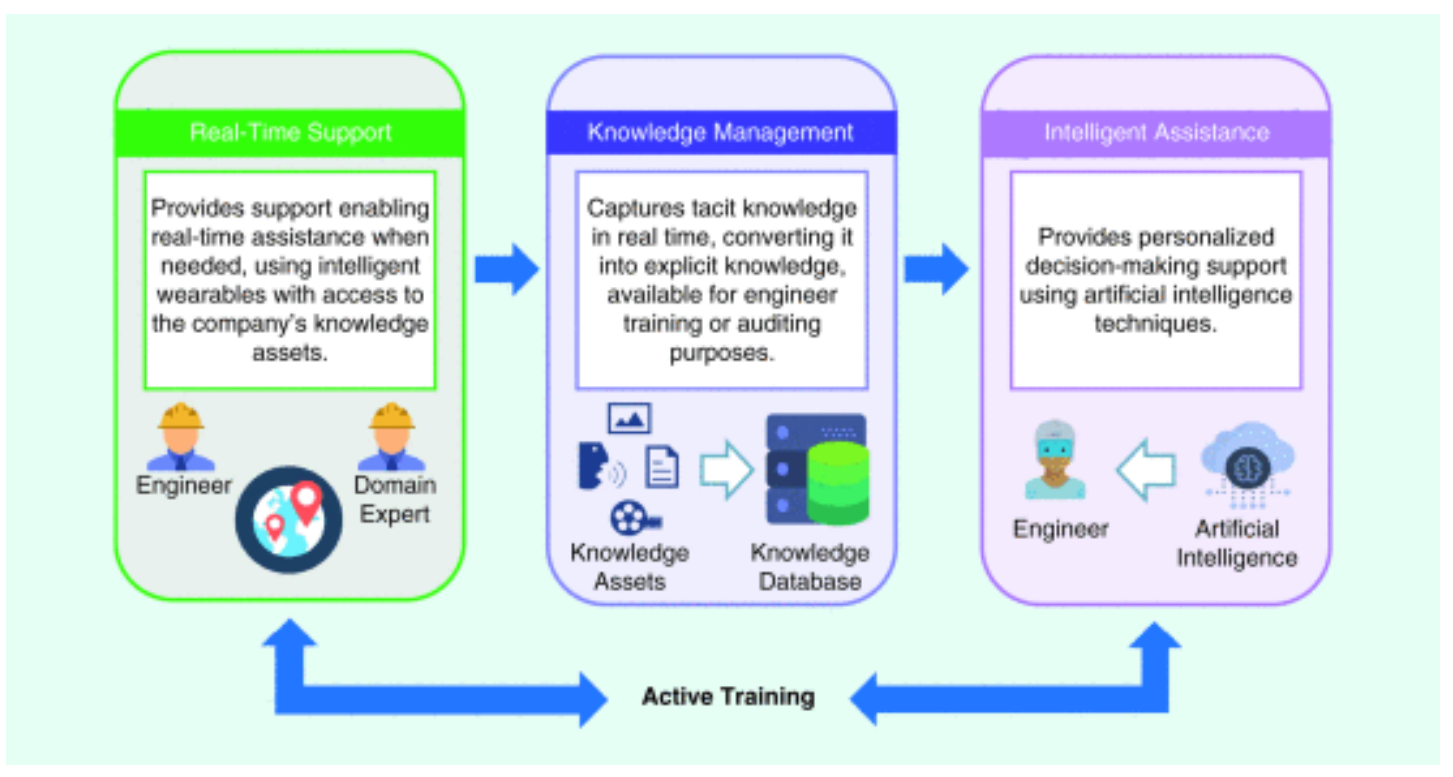

Figure 7. The main benefits of an intelligent immersive system for service operations.

Industries in highly competitive global markets are looking to the application of intelligent immersive systems and applications to reduce training costs, improve worker qualifications, and maximize productivity. AR holds particular promise for maintenance tasks that are uncommon, nonrepetitive, lengthy, or atypical, demonstrating great potential as an effective tool for field service operations. This is because AR has more explicit business-oriented use cases than other technologies. The potential is generated by intelligent wearables ranging from simple reality-assistance glasses to fully immersive smart helmets and holographic displays. However, it is necessary to consider the challenges inherent therein, which include current technology limitations, difficulties in the process of meaningful content creation, incorporation of mechanisms to move from reactive systems to predictive ones, and barriers in new technology adoption within institutions.

Overall, the promise of Service 4.0 resides in the appropriate application of the technology to the business environment, not in the technology itself. Amid all of the great potential, the future value of intelligent immersive environments and systems for on-the-job decision-making support and training depends on how applications are implemented. To determine how best to leverage the potential of such systems, additional evidence-based research demonstrating the efficacy of these applications is necessary. In particular, more field research is needed to enable industries to discern the best applications and implementations for intelligent immersive systems. As the scope of opportunities expands, it presents exciting, groundbreaking possibilities for cutting-edge workplace decision-making support, learning, and training in the 21 st century. 


\section{References}

[1] J. Moar. (2016). Smart glasses: Seeing through the hype. Juniper Research Ltd.

Basingstoke, U.K. [Online]. Available: https://www.juniperresearch.com/ document- library/whitepapers/smart-glasses-\%E2\%80\%93-seeing-through-the-hype

[2] J. P. Gownder. (2017). The enterprise wearables journey. Forrester Research Inc. Cambridge, MA. [Online]. Available: https://www.forrester.com/report/Deliver+Digita I+Operational+Excellence+And+Digital+Customer+Experience+Innovation+With+We arables/-/ERES103381

[3] S. R. Ellis, "What are virtual environments?" IEEE Comput. Graph. Appl. Mag. , vol. 14, no. 1 , pp. 17-22, 1994.

[4] C. Dede, "The evolution of constructivist learning environments: Immersion in dis- tributed, virtual worlds," Educ. Technol. , vol. 35, no. 5, pp. 46-52, Sept. 1995.

[5] M. Slater and S. Wilbur, "A framework for immersive virtual environments (FIVE): Speculations on the role of presence in virtual environments," Teleoperators Virtual Environ ., vol. 6, no. 6, pp. 603-616, Dec. 1997.

[6] W. A . Ijsselsteijn, "History of telepresence," in 3D Videocommunication: Algo - rithms, Concepts and Real-Time Systems in Human Centred Communication, O. Schreer, P. Kauff, and T. Sikora, Eds. Hoboken, NJ: Wiley, 2005, pp. 5-21.

[7] T. P . Caudell and D. W . Mizell, "Augmented reality: An application of heads-up dis- play technology to manual manufacturing processes," in Proc. 25th Hawaii Int. Conf. System Sciences, vol. ii, 1992, pp. 659-669.

[8] M. Wang, V. Callaghan, J. Bernhardt, K. White, and A . Peña-Ríos, "Augmented real- ity in education and training: Pedagogical approaches and illustrative case studies," J. Ambient Intell. Humaniz. Comput. , pp. 1-12, Jul. 2017.

[9] N. Gavish, T. Gutiérrez, S. Webel, J. Rodríguez, M. Peveri, U. Bockholt, and F. Tec- chia, “ Evaluating virtual reality and augmented reality training for industrial mainte- nance and assembly tasks," Interact. Learn. Environ. , vol. 23, no. 6 , pp. 778- 798, 2015.

[10] P. Hořejší, "Augmented reality system for virtual training of parts assembly ," Pro-cedia Eng., vol. 100, pp. 699-706, Jan. 2015.

[11] F. Wild, "The future of learning at the workplace is augmented reality ," Comput. (Long Beach, CA), vol. 49, no. 10, pp. 96- 98, 2016.

[12] Gartner Inc. ( 2016). Gartner's 2016 hype cycle for emerging technologies identi- fies three key trends that organizations must track to gain competitive advantage. Gartner. [Online]. Available: http://www.gartner.com/newsroom/id/3412017

[13] K. Warwick, "Human enhancement: The way ahead," in Proc. Ubiquity Symp.: The Technological Singularity, 2014, pp. $1-7$.

[14] D. Gorecky, M. Schmitt, M. Loskyll, and D. Zuhlke, "Human-machine interaction in the Industry 4.0 era," in Proc. 12th IEEE Int. Conf. Industrial Informatics (INDIN 2014) , pp. 289- 294, 2014.

[15] M. Weiser , "The computer for the 21st century," ACM SIGMOBILE Mobile Com- puting Commun. Rev. , vol. 3 , no. 3 , pp. $3-11,1999$.

[16] J. Steuer, "Defining virtual reality: Dimensions determining telepresence," J. Commun., vol. 42, no. 4 , pp. 73-93, 1992. 
[17] S. Mann, "Mediated reality with implementations for everyday life," Presence: Teleoperators Virtual Environ., vol. 1, Aug. 2002.

[18] M.-S. Yo h , "The reality of virtual reality," in Proc. 7th Int. Conf. Virtual Systems and Multimedia , 2001, pp. 666-674.

[19] M. Heim, Virtual Realism . New York: Oxford Univ. Press, 1998.

[20] R. Schroeder, Possible Worlds: The Social Dynamic of Virtual Reality Technol- ogy . Boulder, CO: Westview , 1996.

[21] G. J. Kim, Designing Virtual Reality Systems: The Structured Approach . London: SpringerVerlag, 2005.

[22] J. H. Lifton, "Dual reality: An emerging medium," Ph.D. dissertation, Massachu- setts Institute Technol., Cambridge, MA, 2007.

[23] P. Milgram and F. Kishino, "A taxonomy of mixed reality visual displays," IEICE Trans. Inform. Syst. , vol. E77-D, no. 12, pp. 1321-1329, 1994.

[24] S. Pastoor and C . Conomis, "Mixed reality displays," in 3D Videocommunication: Algorithms, Concepts and Real-Time Systems in Human Centred Communication, O. Schreer, P. Kauff, and T. Sikora, Eds. Hoboken, NJ: Wiley, 2005, pp. 261- 280.

[25] R. Azuma and R . Azuma, "A survey of augmented reality ," Presence: Teleoperators Virtual Environ. , vol. 6 , no. 4 , pp. 355-385, 1997.

[26] R. Azuma, Y. Baillot, S. Feiner , S. Julier , R. Behringer , and B . Macintyre, "Recent advances in augmented reality," IEEE Comput. Graph. Appl. Mag., vol. 21, no. 6, pp. 34-47, Nov. 2001.

[27] M. Billinghurst, A. Clark, and G. Lee, "A survey of augmented reality," Found. Trends Human-Computer Interact. , vol. 8 , no. 2-3, pp. 73-272, 2015.

[28] E. Bostanci, N. Kanwal, S. Ehsan, and A. F. A. Clark, "User tracking methods for augmented reality ," Int. J. Comput. Theory Eng. , vol. 5 , no. 1 , pp. 93- 98, Feb. 2013.

[29] U.S. National Coordination Office for Space-Based Positioning Naviga- tion and Timing . (2017). GPS accuracy . [Online]. Available: http://www.gps.gov/systems/gps/performance/accuracy/

[30] F. Zhou, H. Been-Lirn Duh, and M. Billinghurst, "Trends in augmented reality tracking, interaction and display: A review of ten years of ISMAR," in Proc. 7th IEEE/ ACM Int. Symp. Mixed and Augmented Reality, 2008, pp. 193-202.

[31] Oxford English Dictionary. (2017). Service: Definition. [Online]. Available: http:// www.oed.com/view/Entry/176678?rskey=E1YkxG\&result=1\#eid

[32] A. K. Dey, "Understanding and using context," Pers. Ubiquitous Comput., vol. 5 , no. 1, pp. $4-7,2001$.

[33] J. E. Aronson, T.-P. Liang, and E. Turban, Decision Support Systems and Intel- ligent Systems. Englewood Cliffs, NJ: Prentice Hall, 2005.

[34] U.S. Air Force Research Laboratory. AFRL autonomy vision: Defense innovation marketplace: Human-machine teaming. [Online]. Available: http://www .defenseinnovationmarketplace.mil/auto_hmt.html

[35] C. E. Billings, Aviation Automation: The Search for a Human-Centered Approach, Boca Raton, FL: CRC, 1997. 
[36] N. Jordan, "Allocation of functions between man and machines in automated systems," J. Appl. Psychol. , vol. 47, no. 3, pp. 161-165, 1963.

[37] P. M . Fitts, "Functions of man in complex systems," Aerosp. Eng. , vol. 21, no. 1, pp. 34- 39, 1962.

[38] J. M. Bradshaw, P. Beautement, M. R. Breedy, L. Bunch, S. V Drakunov, P. J. Fel- tovich, R. R. Hoffman, R. Jeffers, M. Johnson, and S. Kulkarni, "Making agents accept- able to people," in Intelligent Technologies for Information Analysis, 1st Edition, N. Zhong and J. Liu, Eds. Berlin: Springer-Verlag, 2004, pp. 361-406.

[39] P. J. M. Urlings, "Teaming human and machine: A conceptual framework for automa- tion from an aeronautical perspective," Ph.D. dissertation, School of Elect. and Inform. Eng., Div. Inform. Technol., Eng. and Environ., Univ. South Australia, Adelaide, 2003.

[40] M. A. Frigo, E. C. C. da Silva, and G. F . Barbosa, "Augmented reality in aerospace manufacturing: A review ," J. Ind. Intell. Inform. , vol. 4 , no. 2 , pp. 125- 130, 2016.

[41] D. H. Jonassen and T. C. Reeves, "Learning with technology: Using computers as cognitive tools," in Handbook of Research for Educational Communications and Tech-nology D. H. Jonassen, Ed. New York: Simon and Schuster Macmillan, 1996, pp. 694-719.

[42] P. G. Schrader, "Learning in technology: Reconceptualizing immersive environ- ments," AACE J. , vol. 16, no. 4 , pp. 457-475, 2008.

[43] M. Gardner and J. B. Elliott, "The immersive education laboratory: Understand- ing affordances, structuring experiences, and creating constructivist, collaborative processes, in mixed-reality smart environments," EAI Endorsed Trans. Future Intell. Educ. Environ., vol. 1 , no. 1 , pp. $1-13,2014$.

[44] A. Peña-Ríos, H. Hagras, G. Owusu, M. Gardner, G. Owusu, and M. Gardner, "A fuzzy logic based system for mixed reality assistance of remote workforce," in Proc. IEEE Int. Conf. Fuzzy Systems (FUZZ-IEEE) , 2016, pp. 408- 415.

[45] A. Peña-Ríos, H. Hagras, G. Owusu, and M. Gardner , "A fuzzy logic based system for geolocated augmented reality field service support," in IEEE Int. Conf. Fuzzy Systems (FUZZ-IEEE), 2017, pp. 1-6.

[46] $\mathrm{H}$. Hagras, "Embedding computational intelligence in pervasive spaces," Perva- sive Comput. , vol. 6 , no. 3 , pp. 85- 89, 2007.

[47] V. Callaghan, M. Colley, H. Hagras, J. S. Y . Chin, F. Doctor, and G. Clarke, "Programming iSpaces: A tale of two paradigms," in Intelligent Spaces: The Application of Pervasive ICT, 1st ed., S. Wright and A. Steventon, Eds. London: Springer-Verlag, 2006, pp. 389- 421.

[48] H. Hagras, D. Alghazzawi, and G. Aldabbagh, "Employing type-2 fuzzy logic sys - tems in the efforts to realize ambient intelligent environments," IEEE Comput. Intell. Mag. , vol. 10, pp. 4451, Feb. 2015.

[49] L. A. Zadeh, "The concept of a linguistic variable and its application to approxi- mate reasoning-I," Inform. Sci. , vol. 8 , no. 3 , pp. 199-249, 1975.

[50] H. Hagras, "Type-2 FLCs: A new generation of fuzzy controllers," IEEE Comput. Intell. Mag. , vol. 2 , no. 1 , pp. 30- 43, 2007.

[51] J. M. Mendel, Uncertain Rule-Based Fuzzy Logic Systems: Introduction and New Directions . Englewood Cliffs, NJ: Prentice Hall, 2001.

[52] H. Hagras, "A hierarchical type-2 fuzzy logic control architecture for autonomous mobile robots," IEEE Trans. Fuzzy Syst. , vol. 12, no. 4 , pp. 524- 539, 2004. 
[53] H. Hagras and C. Wagner, "Introduction to interval type-2 fuzzy logic controllers: Towards better uncertainty handling in real world applications," IEEE Syst., Man, Cybern. eNewslett. , vol. 27, June 2009.

[54] J. H. Lifton, M. Laibowitz, D. Harry, N. W . Gong, M. Mittal, and J. A. Paradiso, "Metaphor and manifestation: Cross-reality with ubiquitous sensor/actuator networks," Pervasive Comput. , vol. 8 , no. 3 , pp. 24-33, 2009.

[55] A. Peña-Ríos, H. Hagras, G. Owusu, and M. Gardner, "A type-2 fuzzy logic based system for asset geolocation within augmented reality environments," in IEEE Int. Conf. Fuzzy Systems (FUZZ-IEEE ), 2017, pp. 1-6.

[56] P. A. Rauschnabel, A. Brem, and Y. Ro. 2015. "Augmented reality smart glasses: Definition, conceptual insights, and managerial importance." Univ. Michigan-Dear-born, College of Business, Dearborn, 2015.

[57] S. Webel, U. Bockholt, T. Engelke, M. Peveri, M. Olbrich, and C. Preusche, "Aug- mented reality training for assembly and maintenance skills," BIO Web of Conf. , vol. 1, pp. 1-4, Dec. 2011.

[58] Wave Congress, Evolve Media Group. (2017). The opportunities for augmented reality in the enterprise. [Online]. Available: http://www.wavecongress.com/blog/2017- 04-11

The_Opportunities_For_Augmented_Reality_In_The_Enterprise.html

[59] P. Fite-Georgel, "Is there a reality in Industrial Augmented Reality?" in Proc. 10th IEEE Int. Symp. Mixed and Augmented Reality (ISMAR 2011) , 2011, pp. 201-210.

[60] Microsoft Inc. HoloLens: Hardware details. [Online]. Available: https:// developer .microsoft.com/en-us/windows/mixed-reality/hololens_hardware_details

[61] IEEE Standards Association. (2015). Smart glasses roadmap. [Online]. Avail- able: http://standards.ieee.org/about/sasb/iccom/IC15-002-01_Smart_Glasses_Roadmap.pdf

[62] F. Manuri and A. Sanna, "A survey on applications of augmented reality ," Adv. Comput. Sci. , vol. 5 , no. 1 , pp. 18-27, 2016.

[63] B. Bhattacharya and E. Winer, "A method for real-time generation of augmented reality work instructions via expert movements," in Proc. SPIE/IS\&T Electronic Imag- ing (Engineering Reality Virtual Reality), pp. 1-13, 2015.

[64] Domain-Driven Design Community. (2007, Mar. 28). What is domain-driven design? [Online]. Available: http://dddcommunity.org/learning-ddd/what_is_ddd

[65] J. Zhu, S. K. Ong, and A. Y. C. Nee, "An authorable context-aware augmented real- ity system to assist the maintenance technicians," Int. J. Adv. Manuf. Technol. , vol. 66, no. 9-12, pp. 1699-1714, 2013.

[66] M. Anastassova and J. M. Burkhardt, "Automotive technicians' training as a community-ofpractice: Implications for the design of an augmented reality teaching aid," Appl. Ergon., vol. 40, no. 4 , pp. 713-721, 2009.

[67] V. Simón, D. Baglee, S. Garfield, and D. Galar, "The development of an advanced maintenance training programme utilizing augmented reality ," in Proc. 27th Int. Congr. Condition Monitoring and Diagnostic Engineering Management , 2014, pp. 1 -5.

[68] J. M. Spector, "Emerging educational technologies and research directions," Educ. Technol. Soc. , vol. 16, no. 2 , pp. 21-30, 2013.

[69] B. T . Haymes, "The Three-E Strategy for overcoming resistance to technological change," Educause Quarterly, vol. 31, no. 4, pp. 67-69, 2008. 
[70] IEEE Standards Association. (2017). The industry connection program. IEEE Xplore. [Online]. Available: http://standards.ieee.org/develop/indconn/activities.html 